\title{
Control de factores de riesgo cardiovascular en pacientes diabéticos atendidos en dos equipos de Atención Primaria y costes directos asociados. Parte II: Análisis de los costes directos
}

\author{
Grupo de Diabetes de la Sociedad Valenciana \\ DE Medicina familiar y COMUNITARIA SVMFyC \\ A. Bonet Pla, J. Navarro Pérez \\ Médico de Familia. EAP Salvador Pau. Valencia \\ D. Tórtola Graner, A. Collado \\ Médico de Familia. EAP Fuente de San Luis. Valencia \\ V. Gosalbes SOleR \\ Médico de Familia. Técnico de la Unidad Docente de MFyC. Valencia \\ A. Puertas Cabot, J. Sánchez, M. C. Valdivieso Mañas, C. Vivas \\ Residentes de MFyC. Valencia
}

\section{RESUMEN}

Fundamento: la diabetes mellitus ocasiona gas tos elevados, hasta el $5 \%$ del presupuesto sanita rio. Existen algunos estudios de costes que genera la atención a los pacientes diabéticos pero son in completos al no analizar los resultados de las ac tuaciones sanitarias junto a los costes. En nuestro país los datos son aun más escasos.

Objetivo: estudiar el manejo de la diabetes tipo 2 en Atención Primaria, analizando conjuntamen te los resultados según los criterios del Consenso Europeo de 1998 y los costes directos imputados.

Diseño: estudio transversal, en dos Centros de Salud de la ciudad de Valencia.

Pacientes y métodos: estudiamos 513 pacientes de dos centros de salud, atendidos durante 1997. Se valora el grado de control según el Consenso. Como costes directos, se analizan los de la atención profesional de médicos y enfermeras, analíti cas y tratamientos farmacológicos. Se ofrecen cos tes medios globales y por centros, y costes según los diferentes criterios de control tanto globales como por centros.

Resultados: el coste total es de 15.262.668 pese tas. El 77,6\% del gasto lo ocasionan los fármacos, los análisis el 14,3 y el 8,1\% los gastos de
Cardiovascular risk factors control in diabetic pa tients visited in two Primary Health Care teams and associated direct costs. Part II: Direct cost analysis

\section{ABSTRACT}

Background: diabetes care is responsible for al most $5 \%$ of health budget. Studies of cost and out come altogether are scarce, mainly in Spain.

Aim: to study management of type 2 diabetes in Primary Health Care, taking into account health care outcomes upon 1998 European Consensus cri teria and direct costs imputed.

Patiens and methods: we studied 513 patients from two urban health care centers visited in 1997. We colected descriptive independent variables and as dependent variable control degree upon 1998 European Consensus criteria. As directs costs we assessed professional care (doctors and nurses ti me), laboratory test and medications. We present mean patient cost and the mean and total costs of the different control level.

Results: total cost is 15.262 .668 pesetas. $77.6 \%$ of cost is due to prescribed drugs, $14.3 \%$ to labora tory test and $8.1 \%$ to personnel cost. Total mean cost is 29.751 pesetas. Well controled mean cost is 77.475 pesetas. Cost of patient that reach low risk considering the whole CVRF is 1.695 .852 pesetas.

Conclusions: the wide range of cost is a conse quence of the unefficience in the management of diabetes. Our results show how wrong the strategy

Aceptación: 14-06-02 
personal. El coste medio global es de 29.751 pese tas. El coste medio por paciente con buen control glucémico se sitúa en 77.475 pesetas. El coste de cada paciente que alcanza bajo riesgo según todos los criterios es de 1.695.852 pesetas.

Conclusiones: hay una amplia variación de cos tes que refleja la variabilidad en la atención a los pacientes. Nuestros datos señalan que tener en cuenta sólo los costes medios puede inducir a error. Por el contario, al distribuir los gastos ex clusivamente entre los que alcanzan buen control se resalta el peor rendimiento de los recursos.

Palabras clave: Diabetes. Factores de riesgo cardiovascular. Evaluación de resultados. of means costs is. Opposite to it, dividing only cost among those patients well controled enhances unefficiency.

Key words: Diabetes. Cardiovascular risk fac tors. Outcome assessment.

\section{INTRODUCCIÓN}

La diabetes mellitus (DM) es una enfermedad con una elevada prevalencia, principalmente la DM tipo 2 que en pacientes de edad avanzada puede llegar al $10 \%{ }^{1,2}$. La diabetes mellitus ocasiona gastos elevados, cuantificados por algunos autores en el $5 \%$ del presupuesto sanitario global ${ }^{3,4}$ :

Existen estudios que analizan el gasto pormenorizado de la DM tanto tipo 1 como tipo 2, comprobándose cómo la mayor partida de gasto se debe a los gastos indirectos suponiendo éstos el $55 \%{ }^{3}$. Dentro de nuestro país se ha realizado también algún análisis de los costes que genera la atención a los pacientes diabéticos 5,6 . Estos estudios ofrecen únicamente los costes globales distribuidos por conceptos. Por otro lado se han publicado también, estudios que analizan aspectos concretos de la atención sanitaria a los pacientes diabéticos como el tratamiento insulínico o la hospitalización ${ }^{8,9}$.

La mayor parte de todos los trabajos realizados son descripciones de costes ${ }^{10}$ y suponen una valoración económica incompleta al no analizar los resultados de las actuaciones sanitarias junto a los costes. En nuestro país los datos son aun más esca$\operatorname{sos}^{11}$.

El Consenso Europeo de $1998^{12}$, establece unos criterios de buen control que suponen un reto importante para los profesionales sanitarios especialmente para los que trabajan en Atención Primaria. En la primera parte de este trabajo dedicada a conocer los resultados de la atención sanitaria a los pacientes diabéticos, hemos informado de los porcentajes de control de los factores de riesgo cardiovascular (FRCV). Como ya hemos comentado, esos resultados distan mucho de lo que sería deseable. Incorporar los criterios de costes y valorarlos conjuntamente con los criterios del Consenso Europeo ayudaría no sólo a conocer en qué medida la pobla- ción diabética debe ser mejor controlada sino la eficiencia de los recursos empleados.

En este trabajo realizamos una evaluación de los costes directos de la atención sanitaria a una muestra de pacientes diabéticos tipo 2 de dos centros de salud y su relación con los resultados intermedios alcanzados en ellos.

\section{PACIENTES Y MÉTODOS}

El objetivo del presente estudio es realizar un análisis de los resultados medidos en mejoras en el estado de salud (según el Consenso Europeo de 1998) y los costes directos que ello supone en dos Equipos de Atención Primaria (EAP) de la ciudad de Valencia el Centro de Salud Fuente de San Luis y el Centro de Salud Salvador Pau.

Las características de la población y el método ya fueron descritos en la primera parte del estudio. Sólo describiremos aquí lo referente al cálculo de los costes.

A pesar de ser los costes indirectos los que suponen la mayor partida del gasto, debido a lo difícil de conocerlos, sobre todo en un estudio retrospectivo y dado que no sólo tienen implicaciones sanitarias sino también sociales, en el estudio se valoran sólo los costes directos y dentro de ellos, exclusivamente los generados por la atención profesional de médicos y enfermeras, los de las exploraciones analíticas y los debidos a tratamientos farmacológicos, de un año de atención sanitaria. Calculándose:

1. Los costes de la medicación. Estableciendo el precio medio de 1 comprimido de sulfanilureas, biguanidinas e inhibidores de las disacaridasas y multiplicando por el número de comprimidos prescritos de cada uno de los grupos terapéuticos mencionados. Se emplea el mismo sistema para calcular el coste de la insulina. 
2. Los costes de las exploraciones analíticas. A partir de los precios facilitados por el hospital de referencia de cada uno de los centros de salud.

3. Los costes de personal. Determinando una duración media de 15 minutos para una consulta de enfermería y de 5 minutos para una consulta normal ( $\sin$ exploración física) de facultativo y de 15 minutos para una consulta completa de facultativo (con exploración física). A partir de los salarios brutos medios de estos profesionales se cuantifica el precio de la visita.

En el análisis de costes se valoran los costes medios que resultan de dividir los gastos de cada apartado por los pacientes. Para conocer cómo se modifican los costes según los resultados alcanzados se calculan los costes de los diferentes niveles de control, dividiendo los gastos de cada apartado y totales sólo por el número de pacientes que alcanza el objetivo de control analizado. Los costes que se presentan son: a) costes totales y por centros; b) costes totales y por centros de cada uno de los apartados; c) costes medios totales y por centros según la siguiente fórmula costes totales/número pacientes considerados; d) costes medios totales y por centros de cada apartado según la siguiente fórmula: costes apartado/número pacientes considerados; y e) coste medio total y por centros según criterios de control según la siguiente fórmula: coste total/pacientes que alcanzan los diferentes grados de control. Asimismo se realiza un análisis de sensibilidad modifi- cando los costes del apartado de analíticas al calcular el importe total que resultaría de aplicar los costes de otro estudio ${ }^{5}$ y lo mismo en el apartado de costes de personal ${ }^{6}$.

\section{RESULTADOS}

El número total de pacientes estudiados fue de 513. En la primera parte del trabajo se comentan aspectos referidos a la población estudiada.

En la tabla I se presentan los costes totales del programa y por apartados para los dos centros conjuntamente y por separado. Como se observa el coste total del programa es de 15.262 .668 pesetas; el coste de los fármacos es de 11.847 .169 (77,6\%); el de las exploraciones $2.192 .743(14,3 \%)$; el del personal 1.222 .756 pesetas $(8,1 \%)$. Los costes por centros se distribuyen al 53/47\% entre los centros 1 y 2 respectivamente excepto en lo que se refiere a los costes de personal en los que la distribución es $66 / 34 \%$. En la tabla II se recogen los costes medios por paciente totales y por apartados para los dos centros conjuntamente y por separado. El coste medio global es de 29.751 pesetas; por analíticas es de 4.377 ptas; por fármacos 23.065 ptas.; de personal 2.309 ptas. A continuación se recogen los datos separados por centros.

En la tabla III se ofrecen los costes medios por paciente según los diferentes criterios de control. Si

Tabla I

\begin{tabular}{lccc}
\multicolumn{4}{c}{ COSTES TOTALES DEL PROGRAMA } \\
\hline Coste total & Coste fármacos & Exploraciones & Personal \\
\hline $15.262 .668(100 \%)$ & $11.847 .169(77,6 \%)$ & $2.192 .740(14,3 \%)$ & $1.222 .756(8,1 \%)$ \\
\hline Coste Centro 1 & $6.218 .925(52 \%)$ & $1.162 .966(53 \%)$ & $817.155(66 \%)$ \\
\hline $8.199 .047(53 \%)$ & $5.628 .244(48 \%)$ & $1.029 .774(47 \%)$ & $405.603(34 \%)$ \\
\hline Coste Centro 2 & & &
\end{tabular}

Tabla II

COSTES MEDIOS POR PACIENTE GLOBALES Y POR APARTADOS Y SEGÚN CENTROS

\begin{tabular}{lccc}
\hline Coste medio paciente & Coste fármacos & Exploraciones & Personal \\
\hline 29.751 & 23.093 & 4.274 & 2.309 \\
\hline $\begin{array}{l}\text { Coste Centro 1 } \\
28.768\end{array}$ & 21.820 & 4.080 & 2.867 \\
\hline Coste Centro 2 & 24.685 & 4.516 & 1.778 \\
30.980 & & & \\
\end{tabular}


Tabla III

COSTES POR PACIENTE SEGÚN CRITERIOS DE CONTROL

Costes por
paciente

Bajo riesgo $\left(\mathrm{HbA}_{1 \mathrm{c}}<6,5 \%\right)$

77.475

Bajo riesgo+ riesgo macrovascular $\left(\mathrm{HbA}_{1 \mathrm{c}} \geq 6,5<7,5 \%\right)$

48.607

Bajo riesgo $\left(\mathrm{HbA}_{1 \mathrm{C}}+\mathrm{CT}+\mathrm{TA}\right)$

1.695 .852

Bajo riesgo y riesgo $\left(\mathrm{HbA}_{1 \mathrm{C}}+\mathrm{CT}+\mathrm{TA}\right)$

148.181

$\mathrm{HbA}_{1 \mathrm{c}}$ : hemoglobina glicada $\mathrm{A}_{1 \mathrm{c}}$; $\mathrm{CT}$ : colesterol total; TA: tensión arterial

se considera únicamente el control glicémico, el coste medio por paciente con buen control según la hemoglobina glicada $\left(\mathrm{HbA}_{1 \mathrm{C}}<6,5 \%\right)$ se sitúa en 77.475 ptas.; el coste medio por paciente con riesgo macrovascular $\left(\mathrm{HbA}_{1 \mathrm{C}}>6,5<7,5 \%\right)$ es de 48.607 pesetas. Empleando todos los criterios, el coste de los pacientes que alcanzan bajo riesgo es de 1.695.852 ptas. mientras que el coste de los pacientes de bajo riesgo y de riesgo es de 148.181 pesetas.

En la tabla IV se muestran los costes medios por paciente según los diferentes criterios de control por centros. Los costes por paciente con buen control

\section{Tabla IV}

COSTES POR PACIENTE SEGÚN GRADOS DE CONTROL POR CENTROS

\begin{tabular}{lcc}
\hline Grados de control & Centro 1 & Centro 2 \\
\hline $\begin{array}{l}\text { Coste paciente sin riesgo } \\
\left(\mathrm{HbA}_{1 \mathrm{C}}<6,5 \%\right)\end{array}$ & 90.099 & 66.637 \\
$\begin{array}{l}\text { Coste sin riesgo + riesgo } \\
\text { macrovascular } \\
\left(\mathrm{HbA}_{1 \mathrm{C}} \leq 6,5<7,5 \%\right)\end{array}$ & 52.223 & 35.495 \\
$\begin{array}{l}\text { Coste bajo riesgo y } \\
\text { riesgo cardiovascular }\end{array}$ & 1.639 .809 & 1.765 .905 \\
\hline
\end{tabular}

$\mathrm{HbA}_{1 \mathrm{c}}$ : hemoglobina glicada $\mathrm{A}_{1 \mathrm{c}}$ según la hemoglobina glicada $\left(\mathrm{HbA}_{1 \mathrm{C}}<6,5 \%\right)$ de los centros 1 y 2 son respectivamente $90.099 / 66.637$ ptas.; con riesgo macrovascular $\left(\mathrm{HbA}_{1 \mathrm{C}}>6,5<7,5 \%\right)$ son respectivamente de 52.223/35.495 ptas. Empleando todos los criterios, los costes de los pacientes con bajo riesgo y riesgo de los centros 1 y 2 son respectivamente 1.639.809/1.765.905 pesetas.

Finalmente en la tabla V se dan los resultados del análisis de sensibilidad, utilizar las tarifas que ofrece el estudio de Hart et al. ${ }^{5}$ para las analíticas supone un coste de 2.394.684 ptas., si se utilizan los precios del estudio CODE $^{6}$ los costes del apartado de personal se sitúan en 5.535.252 ptas.

\section{DISCUSIÓN}

En primer lugar comentaremos las limitaciones del presente estudio. En lo que se refiere a la población y la muestra, nos remitimos a lo ya expuesto en la primera parte del estudio. En cuanto al tamaño de la muestra, calculado para obtener datos estadísticamente válidos, es por tanto suficiente para el estudio económico.

El tercer tipo de limitación viene dado por el método empleado para realizar el estudio económico, sin embargo por no ser posible llevar a cabo las discusiones acerca de aquéllas sin entrar en aspectos concretos de los resultados dejaremos este aspecto para más adelante.

En primer lugar queremos explicar la estrategia de análisis que hemos llevado a cabo y que es la misma que empleamos en un estudio anterior ${ }^{13} y$ que describimos con más detalle en otro lugar ${ }^{14}$. En esencia nuestro planteamiento se basa en que, aún sabiendo que cualquier actuación sanitaria que mejore una variable continua puede tener una repercusión positiva sobre la salud de las personas, desde un punto de vista de la gestión, no alcanzar unos determinados niveles de efectividad (valorada con los mejores indicadores de los que se disponga en cada caso) supone un gasto parcialmente injustificado que incluso puede llegar a ser considerado absolutamente injustificado y llevarnos a considerar como completamente desperdiciados los recur-

Tabla V

\begin{tabular}{lccc} 
& \multicolumn{3}{c}{ ANÁLISIS DE SENSIBILIDAD } \\
\hline Coste total & Coste fármacos & Exploraciones & Personal \\
\hline $15.262 .668(100 \%)$ & $11.847 .169(77,6 \%)$ & $2.192 .740(14,3 \%)$ & $1.222 .756(8,1 \%)$ \\
Coste total & Coste fármacos & Exploraciones Hart & Personal \\
\hline $15.464 .609(100 \%)$ & $11.847 .169(76,6 \%)$ & $2.394 .684(15,4 \%)$ & $1.222 .756(8,0 \%)$ \\
Coste total & Coste fármacos & Exploraciones & Personal CODE \\
\hline $19.575 .161(100 \%)$ & $11.847 .169(60,5 \%)$ & $2.192 .740(11,3 \%)$ & $5.535 .252(28,2 \%)$ \\
\hline
\end{tabular}


sos empleados para atender a una población. No tener en cuenta este punto de vista supone asumir que cualquier cantidad de recursos que se emplee está justificada, lo que resulta un despropósito.

Ese principio se traduce metodológicamente, en una estrategia de evaluación que fija niveles de exigencia creciente: en un primer nivel, valoración de la relación entre resultados y costes en forma de gastos medios por paciente dividiendo todos los gastos por el total de pacientes estudiados; en un segundo nivel sólo consideramos los gastos medios de los pacientes que alcanzan los diferentes grados de buen control. Para ello dividimos los gastos de cada grupo de control por los pacientes que se encuadran en cada uno de ellos; finalmente el tercer nivel considera todos los gastos y los reparte sólo entre los pacientes que alcanzan determinados niveles de control. Los resultados se dan globalmente para todos los pacientes y separados por centros.

Al aplicar esta metodología se observa cómo los costes por paciente se mueven entre un mínimo de 29.751 ptas. en el supuesto más flexible y un máximo de 1.695.852 ptas. en el supuesto más estricto. Esta amplia variación en los costes no hace más que reflejar rentabilidad del programa realizado y más que su importancia absoluta (que oscilará según el porcentaje de buen control) pone de manifiesto la conveniencia de tener presente siempre un criterio combinado para valorar los resultados de los programas. Esta forma de presentar los costes es especialmente interesante al analizar los resultados por centros; al valorar únicamente los costes medios, el centro 1 tiene unas cifras apreciablemente inferiores (26.544 menos por paciente y año) lo que supondría un ahorro de más de un millón de pesetas para el total de los pacientes; sin embargo al considerar el coste por paciente sin riesgo o sin riesgo más riesgo macrovascular el centro 2 tiene una mejor razón coste resultado alcanzado que oscila entre 281.544 y 200.736 ptas. por paciente y año. En cambio si se considera el coste por paciente con bajo riesgo y riesgo cardiovascular el centro 1 tiene mejor relación coste resultado que se traduce en un ahorro de 1513.152 ptas. por paciente y año.

Como se puede comprobar por los resultados presentados, considerar sólo los costes medios de los pacientes, tanto los globales como los de cada uno de los grupos de control separadamente, nos puede llevar a concluir que las actuaciones tienen una rentabilidad que realmente no tienen. En el caso de que consideremos sólo los costes medios no tenemos en cuenta los gastos, únicamente los comparamos, que es lo que sucede con la mayoría de los estudios realizados. En el supuesto de que sólo demos costes medios por grupos de control (teniendo en consideración los gastos que generan los pacientes de cada grupo) un mayor coste puede ser el resultado de un mejor control y ese aspecto no queda reflejado por el cálculo de los costes medios, eso es lo que ocurre en nuestro estudio donde los costes del centro que controla mejor gasta más por paciente. Por el contrario al considerar poco rentable todo lo que sea alcanzar buen control y distribuir todos los gastos sólo entre los que están bien controlados resalta, tal vez excesivamente, lo que se hace bien y por tanto la mejora de la la rentabilidad de lo gastado. Desgraciadamente nuestro estudio al no haber sido diseñado para determinar las posibles razones de la variabilidad de la práctica no nos permiten discutir con detalle las causas de estas variaciones en los costes.

Al intentar comparar nuestros resultados con datos que se ofrecen en otros estudios ${ }^{15-19}$ nos vemos limitados por las diferencias metodológicas de los estudios que impiden obtener los mismos resultados. En cualquier caso tres trabajos recientes ofrecen cifras referidas a costes que pueden ser de utilidad.

El primer estudio ofrece datos de costes referidos a todos los pacientes diabéticos del estado español. Según estos autores el coste medio por paciente anual (según precios de 1994) es de 63.390 ptas. ${ }^{5}$. De esta cantidad los costes ambulatorios -incluyendo consultas externas- suponían el $42 \%$, los fármacos el $13 \%$, el autocontrol el $4,7 \%$ y los análisis el $10 \%$. En este mismo estudio se estableció el coste de las visitas ambulatorias en 1.350 ptas. De acuerdo con esos datos el coste por paciente ambulatorio sería aproximadamente de 45.000 ptas. (sumando los costes ambulatorios y de fármacos) cantidad ligeramente superior al coste medio que nosotros informamos y que puede ser debida al mayor precio de las visitas y análiticas que fija ese estudio.

El segundo estudio presenta los datos de coste del control de los pacientes diabéticos españoles comparados con los costes de los pacientes diabéticos de varios países europeos ${ }^{6}$. Según este estudio el coste medio por paciente es de 217.158 ptas. El coste ambulatorio es de 55.504 ptas. y el de los fármacos de 92.224 ptas. El coste medio de la visita del médico de familia es de 1.682 ptas, el del especialista de 4.531 ptas. y el de enfermería de 2.076 ptas. Como en el caso anterior los costes medios son claramente superiores a los que nosotros obtenemos. También aquí las tarifas de las visitas se sitúan por encima de las que nosotros hemos estimado, son especialmente discrepantes las asignadas a las visitas de enfermería.

Son llamativos los costes medios de los pacientes atendidos en Atención Primaria si los comparamos con los costes hospitalarios ${ }^{8,9}$, resultando los primeros claramente inferiores. En este sentido los datos publicados recientemente y los obtenidos por nosotros no permitirían seguir manteniendo la afirmación de que los costes de la DM se reparten al 60/40\% entre el hospital y AP.

Finalmente el tercer estudio ${ }^{20}$ ofrece los resultados de un análisis coste efectividad que considera 
dos opciones de tratamiento, uno tradicional y otro intensivo. Los resultados finales valorados fueron muerte o aparición de cualquier tipo de complicación crónica micro o macrovascular. El estudio económico consideró los costes directos y la estrategia seguida fue la de calcular el coste incremental por año libre de eventos. Se comparó la tasa neta de coste y de efectividad del tratamiento convencional y del tratamiento intensivo. El estudio comprueba que el control intensivo aumenta los costes del tratamiento $659 £(580-739 £)$ respecto al tratamiento convencional. Los costes de las complicaciones suponían las mayores partidas de costes, la media por ingreso hospitalario fue de $4.266 £$ en el grupo convencional y $3.494 £$ en el grupo intensivo diferencia media de $772 £(159-1.385 £)$ Esta reducción del $18 \%$ en los costes se debe a una reducción en la estancia media (-1,3 días) y a un menor número de ingresos $(-0,1)$. La aparición de un evento supone también un aumento en los costes extrahospitalarios posteriores de $241 £$ el primer año; $106 £$ el segundo año y $80 £$ el tercer año. Todos los costes fueron inferiores en el grupo intensivo. Al final el grupo intensivo tenía unos costes por complicaciones inferiores al convencional en $957 £$. Al aplicar los estándares las diferencias son mayores $7.871 £$ en el convencional y $8.349 £$ en el intensivo. El coste por año de vida sin evento es de $1.166 £$. Se concluye que los costes incrementados del control intensivo se compensan con los ahorros por menores complicaciones. Este estudio muy amplio y riguroso ofrece datos de gran interés que confirman la eficiencia del control intensivo de la DM en Atención Primaria en la línea de lo propuesto por nosotros respecto a la necesidad de mejorar los resultados. En cuanto a los costes demuestra que los costes sólo sin consideración de los resultados son engañosos.
Al realizar un estudio de sensibilidad modificando nuestros costes en función de las tarifas de las analíticas que ofrece el estudio de Hart ${ }^{5}$ apenas si se aprecian cambios en el coste del programa, en cambio si se utilizan los precios del estudio $\operatorname{CODE}^{6}$ sí que se produce un incremento apreciable de los costes totales. En cualquier caso como ya indicamos creemos que esa estandarización de tarifas enmascara la eficiencia de las diferentes organizaciones.

Como conclusión de nuestro estudio podemos afirmar que los costes por paciente de nuestros centros son más bajos que los que se recogen en los estudios más conocidos; por otro lado hemos encontrado diferencias en los costes debidas a diferencias en los tres apartados analizados, finalmente comprobamos cómo se modifican los costes (aumentando) al introducir la valoración de los resultados de salud. La eficiencia de los programas de tratamiento de la DM especialmente de la tipo 2 ha merecido algunos artículos ${ }^{19,21}$ en algunas revistas de Atención Primaria, estos artículos son revisiones teóricas que ayudan a tener una visión general de lo que puede ser la evaluación económica en el terreno de la diabetes. Pensamos que nuestro trabajo aporta datos interesantes obtenidos de la práctica asistencial que complementan lo expuesto en aquéllos.

\section{CORRESPONDENCIA:}

Álvaro Bonet Pla

Centro de Salud Salvador Pau

Salvador Pau, 42

46021 Valencia

e-mail: bonet_alv@gva.es

\section{Bibliografía}

1. Goday A, Serrano Ríos M. Epidemiología de la diabetes mellitus en España. Revisión crítica y nuevas perspectivas. Medicina Clínica 1994; 102: 306-15.

2. Ascaso J, Almenar F, Borras MJ, Ibañez E, Monsalve V. Plan Integral de Asistencia al paciente diabético de la Comunidad Valenciana. Conselleria de Sanidad y Consumo, 1995.

3. American Diabetes Association. Economic consequences of diabetes mellitus in the US in 1997. Diabetes Care 1998; 21 : 296-309.

4. Nabarro JDN. Diabetes in the United Kingdom: some facts and figures. Diabet Med 1988; 5: 816-22.

5. Hart W, Espinosa C, Rovira J. El coste de la diabetes mellitus conocida en España. Med Clin (Barc) 1997; 109: 289-93.

6. CODE-2: Cost of diabetes in Europe Type 2. SmithKline Beecham Pharmaceuticals, 1999.
7. Costa B, Estopa A, Borras J, Sabate A. Diabetes y farmacoeconomía. Eficiencia de los sistemas disponibles para la inyección de insulina en España. Aten Primaria 1995; 16: 391-6.

8. Pascual JM, Gonzalez C, de Juan S, Sanchez C, Sanchez B, Perez M. Impacto de la diabetes mellitus en los costes de hospitalización. Med Clin (Barc) 1996; 107: 207-10.

9. Monereo S, Pavón I, Vega B, Elviro R. Diabetes Mellitus: aproximación al coste por proceso aplicado al paciente diabetico ingresado. Endocrinología 1997; 44: 319-25.

10. Drumond M, Stoddart G, Torrance G. Métodos para la Evaluación Económica de los Programas de Atención de la Salud. Madrid: Díaz de Santos, 2001.

11. Bonet A, Asensi A, Brotons F, Gálvez C, Riera C, Villalba JM. Efectividad y costes de un programa de control de la diabetes mellitus en un equipo de Atención Primaria. Actas 
de las XIV Jornadas de Economía de la Salud: 1993 junio 810: Santiago de Compostela. Sociedad Española de Economía de la salud, 1993.

12. International Diabetes federation (Europe). Guidelines for Diabetic Care. A Desktop Guide to type 2 Diabetes Mellitus. Brussels: Walter Witrz Druck\&Verlag, 1999.

13. Bonet A, Navarro J. Los costes de los programas de tratamiento de la hipertensión arterial. Cuadernos de Gestión 1999; 5: 13-24.

14. Bonet A. Estudios económicos en Atención Primaria. Manuales de referencia Astra Zeneca. Madrid: Editorial Doyma, 2000.

15. Selby JV, Ray T, Zhang D, Colby C. Excess Cost of medical care for patients with diabetes in a managed care population. Diabetes care 1997; 20: 1396-402.

16. Jönsson B. Diabetes-the cost of illness and the cost of control. Act Med Scan 1983; 671 (Supl.): 19S-27S.
17. Costa B, Arroyo J, Sabaté A. The economics of pharmacotherapy for diabetes mellitus. Pharmacoeconomics 1997; 11 (2): 139-58.

18. Rubio JA, Álvarez J. Costes económicos de la diabetes: revisión crítica y valoración coste eficacia de las estrategias propuestas para su reducción. Atención Primaria 1998; 22: 239-55.

19. Badía X, Ondategui S. Farmacoeconomía del tratamiento de la Diabetes Mellitus. Cuadernos de Gestión 1999; 5: 124-33.

20. Gray A, Raikou M, McGuire A, Fenn P, Stevens R, Cull C, et al. Cost effectiveness of an intensive blood glucose control policy in patients with type 2 diabetes: economic analysis alongside randomised controlled trial (UKPDS 41) BMJ 2000; 320: 1373-8.

21. Leese B. Economic evaluations of type II diabetes. Pharmacoeconomics 1995; 8 (Supl. 1): 23S-27S. 